\title{
Multitemporal 3D Change Detection in Urban Areas Using Stereo Information from Different Sensors
}

\author{
Jiaojiao Tian \\ Remote Sensing Technology Institute \\ German Aerospace Center (DLR) \\ Wessling, Germany \\ Jiaojiao.tian@dlr.de
}

\author{
Peter Reinartz \\ Remote Sensing Technology Institute \\ German Aerospace Center (DLR) \\ Wessling, Germany \\ Peter.Reinartz@dlr.de
}

\begin{abstract}
For a detailed monitoring of cities only very high resolution (VHR) satellite images as well as airborne imagery are able to provide the required accuracy. Especially not well mapped cities and new build up areas and their infrastructure like buildings, streets, bridges and other objects are of interest. Just the 2D information delivered by single images is often not sufficient when dealing with changes. Therefore stereo imagery is of importance to derive the height component which is of high value in analyzing 3D objects. The paper describes 3D change detection methodology if several Digital Surface Models (DSM) from different dates and sensors are available and provides solutions by the joint use of height and spectral information. The subtraction of DSMs, computed from image pairs acquired at different dates, should give direct results about changes. However, when at least one of the DSMs presents artifacts, a simple DSM subtraction often results in the detection of a combination of real and virtual changes. Therefore morphological based post-processing steps are proposed and adapted to different DSM qualities in order to remove artificial changes. In addition to height information, vegetation and shadow masks are generated, which are of great help in reconstructing sharper and more correct building boundaries. The used data are acquired over the city of Munich, Germany. They consist of a time series of stereo images acquired within the years 2005 to 2010 from space borne (IKONOS and Worldview) and airborne (3K-Camera-System) sensors. Evaluation of the proposed approaches in terms of completeness, correctness, overall accuracy, etc is performed, proving its efficiency and relatively high accuracy for different kinds and combinations of stereo images and consequently different DSM qualities
\end{abstract}

Keywords-Optical Stereo Data, DSM, 3D Change Detection, Building, multi-temporal

\section{INTRODUCTION}

Urban area monitoring by using three-dimensional (3D) data is a very important topic in remote sensing application [1]. Several methods in the bibliography address 3D change detection by using the Lidar DSM [2-4]. The matching based DSM generation of stereo data and its application has become a very important task and an increasing interest in both remote sensing and computer science research could be noted recently [1,5-7]. This is partly due to better availability, lower cost, higher time resolution and larger coverage ability. With the height difference images, the changes can already easily be seen from the images [8-9]. But how to locate the real changes and how to highlight the exact size of the changed area in 3 dimensions, especially from satellite data is still a challenge that needs to be solved.

A task is to combine data of different resolutions, generated by different sensor model and performance. All of this will influence the quality of the generated DEMs, which will finally lead to a lot of artificial changes in the extracted change map. In former studies, we tried to classify the real change by analyzing the changed region in the difference image by using shape features [9]. That method works well in commercial and suburban areas, which exhibit large buildings and houses of similar shape, while for the centres of big cities, which have more complicated building structures, it is quite difficult to define a common character of the buildings.

However, for those stereo imagery, which provide additionally multi-spectral images that are acquired at the same time, more information can be used to improve the change detection results. In this paper, the robust difference is adopted to eliminate the noise that is caused by occlusions matching errors and interpolation of the generated DSMs. After that we fuse the mutual information from the pan-chromatic images to refine the change map. In the last step, the combined usage of morphological features and a land cover mask lead to a refinement of the building shapes. This method is tested by using the DSMs that are extracted from IKONOS-2, WorldView-2 and airborne stereo imagery in Munich city area. The results show that the proposed methodology is well suited for purpose of $3 \mathrm{D}$ change detection.

\section{METHOD}

We aim at generating an initial difference map by using the height information from the stereo imagery and then process this difference map with morphological filtering and land cover maps generated by multispectral data. The focus of the research is on noise reduction or the removal of artificial change in the DSM difference images.

The Digital Surface Models (DSMs) that are used in this paper are computed using the Semi-Global Matching (SGM) method [5-6]. A further co-registration between the resulting DSMs is necessary to remove any shift in three dimensions that might exist between the two DSMs. 


\section{A. Robust difference}

The DSMs generated from stereo images exhibit 3D information but not yet on an object level. The accuracy of the generated DSM is very much dependent on the radiometric data quality, the ground sampling distance (GSD) of the sensor data, the convergence angle of the stereo data and the amount of available stereo pairs. Also it cannot be expected that the stereo data from different dates are acquired by the same instrument, so in many cases a robust difference is essential in obtaining a less noised difference image. To cover this problem, many noise reduction methods have been developed [10]. In this research, the robust difference that is already shown in Tian [11] is adopted.

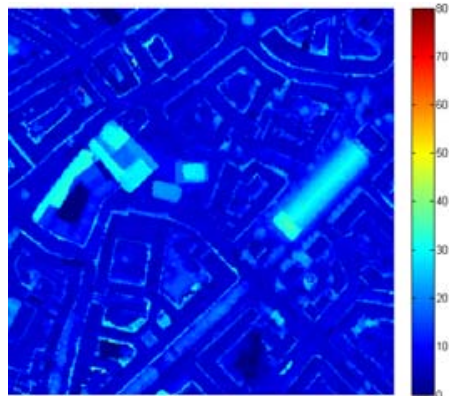

(a)

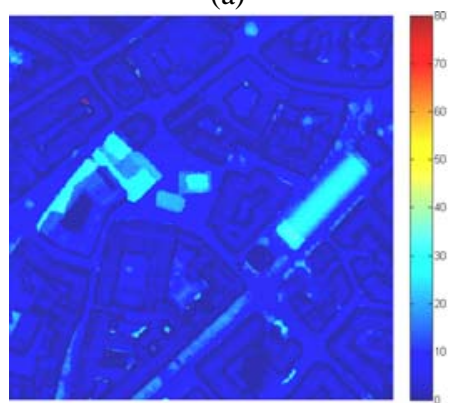

(b)

Figure 1. Comparison of the robust based difference and direct difference

Figure 1 shows a comparison between a simple pixel-topixel difference between two DSMs and a robust DSM difference. In Figure 1(a), although the changed areas are highlighted to some extend, the background is very noisy, which will cause a problem in the change information extraction procedure. In contrast, after executing the robust image difference in Figure 1(b), the noise in the background is successfully reduced, while the bright areas, which are more likely to be real changed areas, are not influenced significantly.

\section{B. Morphological filtering of the change map}

In binary change detections, one of the most important final steps consists in highlighting real positive and negative changes through getting rid of artificial change (noise and other processing errors). Morphological filters which are mathematically based image processing method is adopted to cover this problem. Pesaresi and Benediktsson used it to process remote sensing images [12]. It bases mainly on some basic operation, such as erosion and dilation.

$$
\begin{aligned}
& \operatorname{erode}(x, y)=\min _{\left(x^{\prime}, y^{\prime}\right) \in \text { ker nel }} \operatorname{src}\left(x+x^{\prime}, y+y^{\prime}\right)(1) \\
& \text { dilate }(x, y)=\max _{\left(x^{\prime}, y^{\prime}\right) \in \text { ker nel }} \operatorname{src}\left(x+x^{\prime}, y+y^{\prime}\right)(2)
\end{aligned}
$$

Since our research is focused on changed building extraction and reconstruction, here we defined a square shape as the kernel, src is the cover range of all the pixels in this kernel. An example of the morphological based method is shown in figure 2, figure 2(a) is the original height based thresholding result, many small noises that were produced by the DSM generation procedure are displayed in it. While figure 2(b) is the morphological filter result in which the changed building is well highlighted with sharper edges.

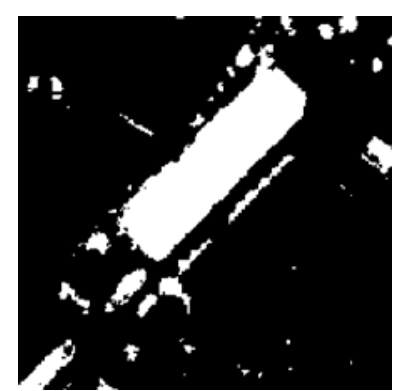

(a)

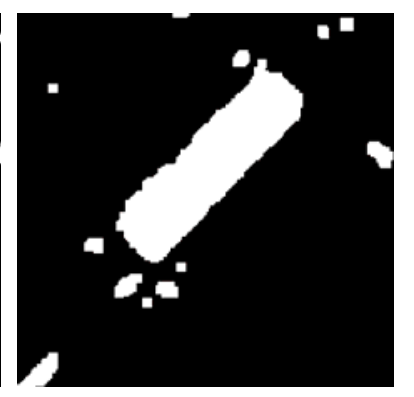

(b)
Figure 2. Morphological based filtering

\section{Fusion with the Land cover maps}

As can be see from figure 2(b) the edges in the lower right side is sharper than the upper left side, by analysing the character of the stereo maps, the side with lower quality of the building border is always located at the shadow parts of the building, as can be see from Figure 3 . The green part represents the real shadow of the building on the ground surface, and the red part is the shadow that is detected from satellite position 1 , while the blue part is the shadow that is detected from satellite position 2. The low DSM quality of the shadow side can be caused by two reasons: i) missing information, as some parts of the red area seen from position 1 are not visible from position 2; ii) the commonly covered parts (blue area) represents still shadow, which has relatively low radiometric values, and will eventually cause matching failures in the DSM generation procedure.

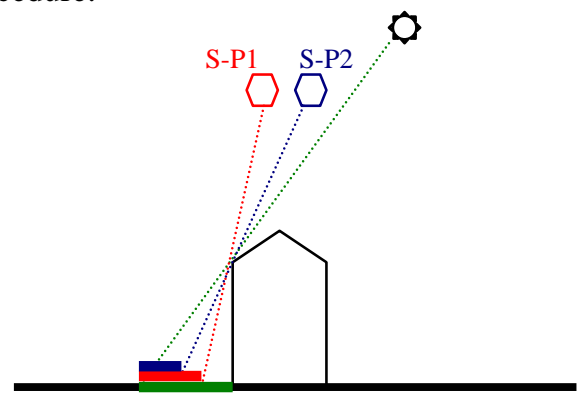

Figure 3. Shadow display in the stereo data 
The existence of shadow can influence the quality of the images, but also can be used to acquire valuable information for building extraction [13]. It is a fact that the shadow parts which have relatively bad matching quality are parallel to the building edges if no other high objects are within the shadow cast. Figure 4 displays the whole procedure of the shadow mask based building shape enhancement. The black line is the original detected edges, and the blue line presents the dilated boundary, which will interact with the shadow mask, which is extracted with the method mentioned by Makarau [14]. The green rectangular shows the refined building shape, further erosion is processed based on the result to keep the original building size. The red polygon represents the refined building shapes.

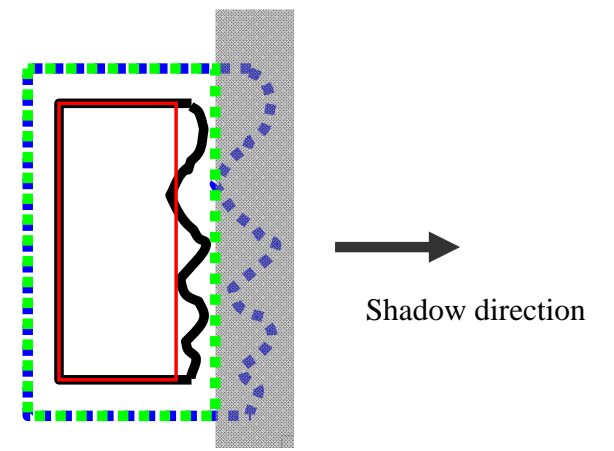

Figure 4. Changed building mask and shadow mask

\section{DATASET}

\section{A. Description of the data}

In order to evaluate the performance of our approach, the data sets acquired over the city of Munich, Germany consist of a time series of stereo images acquired within the years 2005 to 2010 from space borne (IKONOS, 2006 and Worldview, 2010) and airborne (3K-Camera-System, 2007, 2008) sensors are used in our experiments.

- $\quad$ DSMs from satellite stereo imagery.

The IKONOS DSM (called in the following as IKONOS-DSM) were computed from IKONOS in-orbit stereo imagery (level $1 \mathrm{~A}$, viewing angles $+9.25^{\circ}$ and $-4.45^{\circ}$ ) with one meter ground sampling distance (GSD), acquired in July 2005. It has been generated using the Semi Global Matching (SGM) algorithm implemented at DLR [5]. The WorldView-2 data which were acquired in July 2010 have 0.5 meter GSD and the DSM has been generated with the same technology.

- $\quad$ Airborne stereo imagery:

The airborne stereo imagery that are used in our test were acquired by using a new low-cost camera system (name 3Kcamera), which has been build up by the German Aerospace Center (DLR), the GSD for the data is around 0.25 meter. The DSM (called 3K-DSM) is generated with the stereo imagery that were obtained in 2007.

\section{- $\quad$ LiDAR data}

To evaluate the quality of our DSMs, the LiDAR point cloud data acquired in February 2003 (called in the following as LiDAR-DSM) is adopted as a reference data for comparison to the various sources of DSMs. In our experiment, we also generate a change detection mask by comparing the LidarDSM and WorldView-DSM.

\section{B. DSM quality assessment}

The DSM quality assessment is done by comparing them with the LiDAR-DSM. First, several unchanged buildings are chosen from both the IKONOS-DSM, WorldView-DSM and $3 \mathrm{~K}-\mathrm{DSM}$ to look at the different DSM qualities. Figure 5 shows a profile for the comparison of the Lidar-DSM (Blue), WorldView-DSM (Red), IKONOS-DSM (Green) and 3KDSM (Yellow) in a no changed area with several buildings. The maximum errors, minimum errors, bias and standard deviation is calculated to express the quality of the DSM. The resulting figures are showed in Table 1.

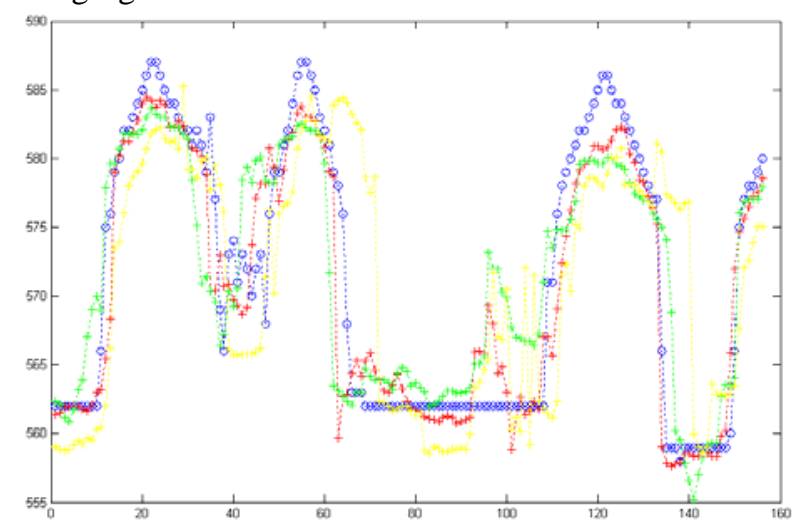

Figure 5. Quality assessment (blue: Lidar-DSM; Red: WorldView-DSM, Green: IKONOS-DSM; Yellow: 3K-DSM )

TABLE I. QUALITY ASSEMMENT FOR PROFILE IN FIG. 5

\begin{tabular}{|c|c|c|c|c|c|}
\hline & $\begin{array}{c}\text { Source } \\
\text { Image }\end{array}$ & $\begin{array}{c}\text { Min } \\
{[\mathbf{m}]}\end{array}$ & $\begin{array}{c}\text { Max } \\
{[\mathbf{m}]}\end{array}$ & $\begin{array}{c}\text { Bias } \\
{[\mathbf{m}]}\end{array}$ & $\begin{array}{c}\text { Std } \\
{[\mathbf{m}]}\end{array}$ \\
\hline 1 & IKONOS & 0.015 & 15.534 & 3.535 & 3.269 \\
\hline 2 & WorldView & 0.017 & 18.357 & 2.382 & 2.668 \\
\hline 3 & $3 \mathrm{~K}$ & 0.002 & 20.211 & 5.282 & 4.737 \\
\hline
\end{tabular}

\section{RESUlts}

\section{A. Expriments}

The experiments are performed by cross comparison of all datasets described in Chapter III. 3D co-registration is firstly done to remove the shift in three dimensions between the DSMs, the same shift values are also applied to the original satellite images. Figure 6 shows the test area we used in the evaluation. Figure $6(\mathrm{a}-\mathrm{c})$ show the panchromatic images of the test area, while (d-f) represent the DSMs. 


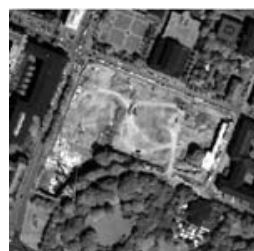

(a)

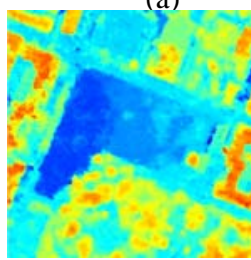

(d)

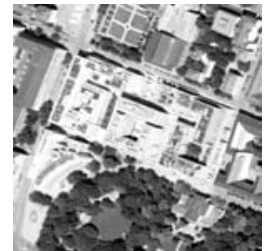

(b)

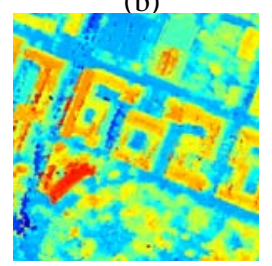

(e)

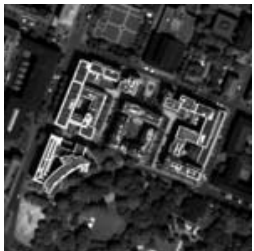

(c)

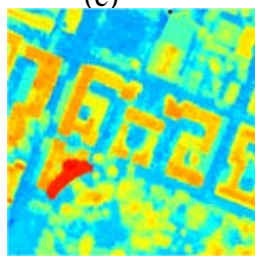

(f)

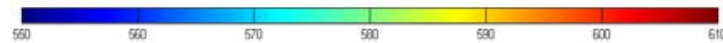

Figure 6. Test data (a: IKONOS-pan; b: 3K camera-pan; c: World View-pan; d: IKONOS-DSM; e:3K-DSM, f. WorldView-DSM)

\section{B. Results and Evaluation}

Two groups of test result are obtained in our experiments. Test 1 are the change detection result by comparing the WorldView (year 2010) dataset with the IKONOS imagery (year 2005), while test 2 shows the change detection results by using the 3K imagery (year 2007) and the IKONOS imagery. The values in Table II give the results based on the number of pixels of false detected change (false alarm), and on the number of not detected change (for missed alarm) divided by the real number of pixels of the changed area using a manually extracted ground truth of building changes.

TABLE II. Result EVAluation AT PIXEL LEVEL IN \%

\begin{tabular}{|c|c|c|c|c|}
\hline & Source Image & $\begin{array}{c}\text { False } \\
\text { alarm }\end{array}$ & $\begin{array}{c}\text { Missed } \\
\text { alarm }\end{array}$ & $\begin{array}{c}\text { Kappa } \\
\text { Coefficient }\end{array}$ \\
\hline \multirow{3}{*}{ Test1 } & Direct-Diff. & 0.35 & 0.10 & 0.77 \\
\cline { 2 - 5 } & Robust-Diff. & 0.25 & 0.11 & 0.80 \\
\cline { 2 - 5 } & $\begin{array}{c}\text { Morphologic- } \\
\text { Refinement }\end{array}$ & 0.22 & 0.12 & 0.81 \\
\hline \multirow{3}{*}{ Test2 } & Direct-Diff. & 0.64 & 0.11 & 0.65 \\
\cline { 2 - 5 } & Robust-Diff. & 0.48 & 0.13 & 0.70 \\
\cline { 2 - 5 } & $\begin{array}{c}\text { Morphologic - } \\
\text { Refinement }\end{array}$ & 0.35 & 0.18 & 0.72 \\
\hline
\end{tabular}

\section{CONCLUSION}

In this paper, a 3D change detection approach based on multitemporal DSMs generated by optical stereo image matching is proposed and evaluated to detect building changes in the city centre of Munich by using 3 kinds of DSMs.

The whole procedure is divided into 3 steps. First, we generate and co-register DSMs acquired at different epochs. Then, we compute the "robust difference images" in order to reduce the noise coming from the different nature of the DSMs used. In fact, the random variations of the stereo image acquisition conditions as well as the blunders caused during the automatic matching and DEM generation process makes urban structures look different from one DSM to another, especially for building walls and edges. As our focus in this paper is the monitoring of urban changes, noise reduction is essential. After that, we generate the change map with both vertical and horizontal change information. To overcome the poor quality of some of the DSM, we refine the changed buildings to rectangular shapes. To confirm the validity of our approach, we compare our results with manual extracted ground truth figures.

It has been shown that DSMs generated from optical stereo imagery can be a reliable source for efficient 3D change detection. The promoted robust change detection method can handle the partly low quality of the DSMs.

\section{REFERENCES}

[1] T. Krauss, P. Reinartz, and U. Stilla, "Extracting Orthogonal Building Objects in Urban Areas From High Resolution Stereo Satellite Image," Pairs, International Archives of Photogrammetry, Remote Sensing and Spatial Information Sciences, 36(3/W49B), 2007.

[2] H. Murakami, "Change Detection of Buildings Using an Airborne Laser Scanner,” ISPRS Journal of Photogrammetry \& Remote Sensing, Vol. 54, pp.148-152, 1999.

[3] X. Huang, L. Zhang, and W. Gong, 'Information fusion of aerial images and LIDAR data in urban areas: vector-stacking, re-classification and post-processing approaches', International Journal of Remote Sensing, 32 (1), pp. 69- 84, 2011

[4] P. Gamba,and B. Houshmand, "Joint analysis of SAR, LIDAR and aerial imagery for simultaneous extraction of land cover, DTM and 3D shape of buildings.” International Journal of Remote Sensing, 23, pp. 44394450, 2002.

[5] P. d'Angelo, M. Lehner, and T. Krauss, “Towards Automated DEM Generation from High Resolution Stereo Satellite Images,” International Society for Photogrammetry and Remote Sensing, pp. 1137-1342, 2008.

[6] H. Hirschmüller, "Stereo processing by semiglobal matching and mutual information," IEEE Transactions on Pattern Analyses and Machine Intelligence, 30 (2), Feb. 2008.

[7] P. Reinartz, R. Müller, M. Lehner, and M. Schroeder, "Accuracy Analysis for DSM and Orthoimage Derived from SPOT HRS Stereo Data Using Direct Georeferencing,” ISPRS Journal of Photogrammetry and Reomote Sensing, Vol. 60 (3), pp. 160-169, 2006.

[8] A. Alobeid, K. Jocabsen, C. Heipke and M. Al Rajhi, "Building Monitoring with Differential DSMS”, ISPRS Hannover Workshop 2011.

[9] J. Tian, H. Chaabouni-Chouayakh, and P. Reinartz, “3D building change detection from high resolution spaceborne stereo imagery. “ Intern. Workshop on Multi-Platform/multi-Sensor Remote sensing and Mapping, M2RSM, Xiamen, China, IEEE Xplore, 2011

[10] L. Bruzzone, and R. Cossu, "An adaptive approach to reducing registration noise effects in unsupervised change detection.” IEEE Transactions on Geoscience and Remote Sensing, Vol. 41 (11), November 2003.

[11] J. Tian, H. Chaabouni-Chouayakh, and P. Reinartz, T. Krauss, and P. d'Angelo, "Automatic 3D change detection based on optical satellite stereo imagery,” ISPRS TC VII Symposium-100 Year ISPRS, Vienna, Austria, July 5-7, 2010.

[12] M. Pesaresi and J. A. Benediktsson, "A new approach for the morphological segmentation of high-resolution satellite imagery,” IEEE Trans.Geosci. Remote Sens., vol. 39 (2), pp. 309-320, Feb. 2001.

[13] B. Sirmacek, and C. Unsalan, “A Probabilistic Framework to Detect Buildings in Aerial and Satellite Images", IEEE Transactions on Geoscience and Remote Sensing, 49 (1), pp. 211-221, 2011.

[14] A. Makarau, R. Richter, R. Müller and P. Reinartz, “Adaptive shadow detection using a blackbody radiator model," IEEE Trans.Geosci. Remote Sens., vol. 49 (6), pp. 2049-2059, 2011 